\title{
Sur les intégrales attachées aux formes automorphes.
}

\author{
Par Goro SHIMURA
}

(Reçu le 24 avril, 1959)

\begin{abstract}
Dans un travail récent [1] M. Eichler a donné une théorie des intégrales attachées aux formes automorphes d'une variable, et en l'appliquant aux correspondances modulaires, il a obtenu la formule de la trace. Si le degré des formes est 2, les formes paraboliques ne sont autres que les formes différentielles de première espèce; et l'on peut définir la variété jacobienne au moyen des périodes des intégrales. Il est à souhaiter d'obtenir l'analogue pour l'intégrale d'Eichler de degré $>2$. L'objet du présent mémoire est de démontrer que, dans le «cas arithmétique», on peut construire, pour chaque entier pair positif $n$, une variété abélienne à partir des formes paraboliques
\end{abstract} de degré $n$.

Nous considérerons l'intégrale d'une certaine forme différentielle vectorielle attachée à une forme parabolique, qu'Eichler a déjà signalée dans [1]; les périodes d'une telle intégrale définissent un cocycle par rapport à une représentation tensorielle $M$ du groupe fuchsien. Si la représentation $M$ est à coefficients entiers, on peut distinguer les formes paraboliques dont les périodes ont des parties réelles entières; celle-ci forment un lattice $D$ dans l'espace $S$ des formes paraboliques. Nous verrons que la partie imaginaire de la métrique de Petersson prend des valeurs rationnelles sur $D$, ce qui démontre que le tore complexe $S / D$ a une structure de variété abélienne. Les opérateurs de Hecke peuvent alors être regardés comme des endomorphismes de la variété abélienne. On devra observer que si les coefficients de $M$ ne sont pas entiers, il n'y a aucune méthode canonique pour définir un tel lattice $D$ pour lequel $S / D$ devienne une variété abélienne, tandis que la formule de la trace pour les opérateurs de Hecke est obtenue dans le cas général. Du moins, cette variété abélienne $S / D$ est sans doute une des choses dont on aura besoin lorsqu'on cherchera à approfondir la théorie arithmétique des formes automorphes.

Le lecteur s'apercevra de la connexion de notre étude avec les théories de A. Weil [6] et de E. Hecke [3]; on pourrait les regarder comme des cas particuliers des intégrales des formes différentielles à valeurs dans un espace fibré vectoriel auquel est fixée une forme hermitienne invariante par le groupe de fibre, dont on rencontre des exemples aussi dans le cas des formes automorphes de plusieurs variables; mais on ne s'en occupera pas dans ce mémoire. 
Notations. On désignera par $\boldsymbol{Z}, \boldsymbol{Q}, \boldsymbol{R}$ et $\boldsymbol{C}$ respectivement l'anneau des entiers rationnels, les corps des nombres rationnels, réels et complexes; $\boldsymbol{R}^{n}, \boldsymbol{C}^{n}$ désignent alors les espaces vectoriels de dimension $n$ sur $\boldsymbol{R}, \boldsymbol{C}$, respectivement, dont on considère les élements comme des matrices à $n$ lignes et une colonne; $\mathrm{GL}(2, \boldsymbol{R})$ désigne le groupe formé des matrices inversibles de degré 2 à coefficients réels, $\mathrm{GL}_{+}(2, \boldsymbol{R})$ le groupe des éléments de $\mathrm{GL}(2, \boldsymbol{R})$ à determinant positif, $\operatorname{SL}(2, \boldsymbol{R})$ le groupe des éléments de $\mathrm{GL}(2, \boldsymbol{R})$ à determinant 1 , et $\operatorname{SL}(2, \boldsymbol{Z})$ le groupe des éléments de $\operatorname{SL}(2, \boldsymbol{R})$ à coefficients entiers. On désignera par ${ }^{t} X$ la transposée d'une matrice $X$, et par $I_{n}$ la matrice unité de degré $n$. Si $z$ est un nombre complexe ou une fonction à valeurs complexes ou une forme différentielle à valeurs complexes, etc., on indiquera par $\bar{z}, \operatorname{Re}(z)$ et $\operatorname{Im}(z)$ le conjugué complexe, la partie réelle et la partie imaginaire de $z$; l'unité imaginaire $\sqrt{-1}$ sera notée par $i$.

\section{§ 1. Les représentations tensorielles de $G L(2, R)$.}

Soit $\left(\begin{array}{l}u \\ v\end{array}\right)$ un vecteur de $\boldsymbol{R}^{2}$; on désignera par $\left(\begin{array}{c}u \\ v\end{array}\right)^{n}$, pour chaque entier positif $n$, le vecteur de $\boldsymbol{R}^{n+1}$ dont les composantes sont $u^{n}, u^{n-1} v, \cdots, u v^{n-1}, v^{n}$. Nous conviendrons d'entendre par $\left(\begin{array}{c}u \\ v\end{array}\right)^{0}$ le nombre 1 . Soit $\sigma$ un élément de $\mathrm{GL}(2, \boldsymbol{R})$; posons

$$
\left(\begin{array}{c}
u_{1} \\
v_{1}
\end{array}\right)=\sigma\left(\begin{array}{c}
u \\
v
\end{array}\right)
$$

On définit, pour chaque entier non-négatif $n$, une matrice réelle $M_{n}(\sigma)$ de degré $n+1$ par

$$
\left(\begin{array}{c}
u_{1} \\
v_{1}
\end{array}\right)^{n}=M_{n}(\sigma)\left(\begin{array}{c}
u \\
v
\end{array}\right)^{n}
$$

On voit que $M_{n}$ donne une représentation de $\operatorname{GL}(2, \boldsymbol{R})$, pour chaque $n .\left(\begin{array}{c}w \\ z\end{array}\right)$ étant un autre vecteur de $\boldsymbol{R}^{2}$, posons

On a evidemment

$$
\left(\begin{array}{c}
w_{1} \\
z_{1}
\end{array}\right)=\sigma\left(\begin{array}{c}
w \\
z
\end{array}\right)
$$

$$
\left(u_{1} z_{1}-v_{1} w_{1}\right)^{n}=(\operatorname{det} \sigma)^{n}(u z-v w)^{n} .
$$

Définissons une matrice $P_{n}$ de degré $n+1$ par

$$
(u z-v w)^{n}={ }^{t}\left(\begin{array}{c}
u \\
v
\end{array}\right)^{n} P_{n}\left(\begin{array}{c}
w \\
z
\end{array}\right)^{n} .
$$

On a alors, pour tout $n$ et pour tout $\sigma \in \mathrm{GL}(2, \boldsymbol{R})$ : 
et

$$
\begin{gathered}
{ }^{t} M_{n}(\sigma) P_{n} M_{n}(\sigma)=(\operatorname{det} \sigma)^{n} P_{n}, \\
{ }^{t} P_{n}=(-1)^{n} P_{n}
\end{gathered}
$$

$$
M_{n}\left(-I_{2}\right)=(-1)^{n} I_{n+1}
$$

Par suite, si $n$ est pair, $M_{n}$ donne une représentation du groupé quotient $\mathrm{GL}(2, \boldsymbol{R}) /\left\{ \pm I_{2}\right\}$.

\section{§ 2. La métrique de Petersson.}

On désigne par $\mathscr{H}$ le demi-plan complexe $\operatorname{Im}(z)>0$, où $z$ est une variable complexe. Pour chaque élément $\sigma=\left(\begin{array}{ll}a & b \\ c & d\end{array}\right)$ de $\mathrm{GL}_{+}(2, \boldsymbol{R})$, on définit une transformation $z \rightarrow \sigma(z)$ de $\mathscr{H}$ par

$$
\sigma(z)=\frac{a z+b}{c z+d}
$$

Alors, le groupe des transformations analytiques de $\mathscr{H}$ est isomorphe au groupe $\operatorname{SL}(2, \boldsymbol{R}) /\left\{ \pm I_{2}\right\}$. On posera

$$
J(\sigma, z)=(c z+d)^{-1}
$$

On voit facilement qu'on a

$$
J(\sigma \tau, z)=J(\sigma, \tau(z)) J(\tau, z)
$$

pour tout $\sigma, \tau \in \mathrm{GL}_{+}(2, \boldsymbol{R})$. Avec les notations du $\S 1$, on a

$$
\left(\begin{array}{c}
\sigma(z) \\
1
\end{array}\right)^{n}=J(\sigma, z)^{n} M_{n}(\sigma)\left(\begin{array}{c}
z \\
1
\end{array}\right)^{n}
$$

Soit $G$ un sous-groupe de $\operatorname{SL}(2, \boldsymbol{R})$. On supposera dans ce qui suit, que $G$, comme groupe de transformations de $\mathscr{H}$, est un groupe fuchsien, c'est à dire que $G$ est un sous-groupe discret de $\operatorname{SL}(2, \boldsymbol{R})$ pour lequel $\mathscr{K} / G$ ait une mesure finie. Soit $m$ un entier positif. On entendra par une forme parabolique de degré $m$ par rapport à $G$, comme d'ordinaire, une fonction $f$ sur $\mathscr{G}$ jouissant des propriétés (P1-3) suivantes:

(P1) $f$ est holomorphe sur $\mathcal{H}$;

(P2) $f(\sigma(z)) J(\sigma, z)^{m}=f(z)$ pour tout $\sigma \in G$.

Pour exprimer la condition (P3), considérons un point parabolique $s$ de $G ; s$ est un nombre réel ou le point à l'infini $\infty$. Posons

$$
\rho=\left(\begin{array}{ll}
-s & 1 \\
-1 & 0
\end{array}\right) \text { ou } \rho=I_{2}
$$

selon que $s$ est un nombre réel ou $\infty$. Alors chaque transformation de $G$ ayant $s$ comme point fixe est une puissance $\tau^{k}$ d'un élément 


$$
\tau=\rho\left(\begin{array}{cc}
1 & h \\
0 & 1
\end{array}\right) \rho^{-1}
$$

de $G$, où $h$ est un nombre positif. Posons

$$
q=\exp \left(2 \pi i h^{-1} z\right)
$$

Alors, la condition (P3) s'énonce:

(P3) Pour tout point parabolique $s$ de $G$, la fonction $f(\rho(z)) J(\rho, z)^{m}$ est holomorphe comme fonction de $q$ et nulle en $q=0$.

On désignera par $S_{m}(G)$ l'espace linéaire des formes paraboliques de degré $m$ par rapport au groupe $G$. Dans ce mémoire, nous ne nous occuperons que des formes paraboliques de degré pair.

D'après Petersson [4], on peut introduire comme suit une métrique dans l'espace $S_{m}(G)$. Posons d'abord

$$
d v=\frac{i}{2 y^{2}} d z d \bar{z}=\frac{1}{y^{2}} d x d y,
$$

où $z=x+i y$; $d v$ est alors une forme différentielle invariante par toute transformation de $\mathrm{SL}(2, \boldsymbol{R})$. On définit le produit scalaire $(f, g)$ de deux éléments $f, g$ de $S_{m}(G)$ par

$$
(f, g)=\int_{\mathscr{T}^{\prime}} f(z) \overline{g(z)} y^{m} d v
$$

où $\mathscr{I}$ est un domaine fondamental quelconque de $G$; on sait que cette intégrale converge pour tout $f, g \in S_{m}(G)$ et $(f, g)$ est une forme hermitienne positive non-dégénérée.

Considérons maintenant la forme différentielle vectorielle $d_{\delta_{n}}$ sur $\mathscr{T}$ définie par

$$
d_{z_{n}}=\left(\begin{array}{c}
z \\
1
\end{array}\right)^{n} d z=\left(\begin{array}{c}
z^{n} d z \\
z^{n-1} d z \\
\vdots \\
z d z \\
d z
\end{array}\right) .
$$

On a alors, en vertu de (3), pour tout $\sigma \in \mathrm{GL}_{+}(2, \boldsymbol{R})$,

$$
d_{\mathfrak{z}_{n}} \circ \sigma=(\operatorname{det} \sigma) J(\sigma, z)^{n+2} M_{n}(\sigma) d_{\mathfrak{z}_{n}},
$$

$\omega \circ \sigma$ désignant la transformée de la forme différentielle $\omega$ par la transformation $\sigma$. Si $f(z)$ est un élément de $S_{n+2}(G)$, on a

$$
\left(f d_{z_{n}}\right) \circ \sigma=M_{n}(\sigma) f d_{i n}
$$

pour chaque $\sigma \in G$. Soit $g$ un autre élément de $S_{n+2}(G)$. On voit, compte tenu de la relation (1), que la forme différentielle

$$
{ }^{t}\left(f d_{b_{n}}\right) P_{n}\left(\overline{\left.g d_{j_{n}}\right)}\right.
$$


est invariante par le groupe $G$. On vérifie aisément

$$
{ }^{t}\left(f d_{z_{n}}\right) P_{n}\left(\overline{g d d_{z_{n}}}\right)=-(2 i)^{n+1} f \cdot \bar{g} \cdot y^{n+2} d v
$$

il en résulte qu'on a

$$
-(2 i)^{n+1}(f, g)=\int_{\mathscr{F}} t\left(f d z_{n}\right) P_{n}\left(\overline{g d z_{n}}\right) .
$$

Posons, pour deux éléments $f, g$ de $S_{n+2}(G)$,

$$
\Lambda(f, g)=2^{n-1} i[(f, g)-(g, f)] \text {. }
$$

On vérifie facilement que $\Lambda(f, g)$ est une forme $\boldsymbol{R}$-bilinéaire alternée et $\Lambda(f, i g)$ est une forme symétrique positive non-dégénérée.

Nous aurons à nous servir par la suite de la partie réelle $\operatorname{Re}\left(f d_{z_{n}}\right)$ de la forme différentielle $f d_{z_{n}}$. Comme $M_{n}(\sigma)$ est une matrice réelle, on a

$$
\operatorname{Re}\left(f d_{z_{n}}\right) \circ \sigma=M_{n}(\sigma) \operatorname{Re}\left(f d_{z_{n}}\right)
$$

pour tout $\sigma \in G$; par suite, la forme ${ }^{t} \operatorname{Re}\left(f d_{z_{n}}\right) P_{n} \operatorname{Re}\left(g d_{z_{n}}\right)$ est invariante par $G$. Supposons que $n$ soit un entier pair non-négatif; la matrice $P_{n}$ est alors symétrique; on obtient donc

$$
4{ }^{t} \operatorname{Re}\left(f d_{z_{n}}\right) P_{n} \operatorname{Re}\left(g d_{z_{n}}\right)={ }^{t}\left(f d_{z_{n}}\right) P_{n}\left(\overline{g d_{z_{n}}}\right)-{ }^{t}\left(g d_{z_{n}}\right) P_{n}\left(\overline{\left.f d_{z_{n}}\right)} .\right.
$$

D'après la formule (6), $\Lambda(f, g)$ s'exprime sous la forme

$$
\Lambda(f, g)=(-1)^{\frac{n}{2}+1} \int_{\mathscr{I}}{ }^{t} \operatorname{Re}\left(f d_{z_{n}}\right) P_{n} \operatorname{Re}\left(g d_{z_{n}}\right) .
$$

\section{§ 3. Groupes de cohomologie.}

$G$ étant comme ci-dessus, désignons par $Y$ l'ensemble de toutes les transformations paraboliques de $G$. Soit $M$ une représentation de $G$ par des matrices de degré $k$ à coefficients réels. On va définir un certain groupe de cohomologie attaché à $M$. Soit x une application de $G$ dans $\boldsymbol{R}^{k}$; on appelle $\mathfrak{x}$ un cocycle parabolique par rapport à $M$ s'il satisfait aux conditions suivantes :

(C1) $\mathfrak{x}(\sigma \tau)=\mathfrak{x}(\sigma)+M(\sigma) \mathfrak{x}(\tau)$ pour tout $\sigma, \tau \in G$;

(C2) pour chaque $\tau \in Y$, il existe un vecteur $\mathfrak{a} \in \boldsymbol{R}^{k}$ tel que l'on ait $\mathfrak{x}(\tau)=$ $\mathfrak{a}-M(\tau) \mathfrak{a}$; le vecteur $\mathfrak{a}$ peut dépendre de $\tau$.

On désignera par $Z(M, \boldsymbol{R})$ l'espace linéaire des cocycles paraboliques par rapport à $G$. On voit facilement que si x satisfait à la condition $(\mathrm{C} 1)$, on a

$$
\begin{gathered}
\mathfrak{x}(1)=0, \\
\mathfrak{x}\left(\sigma^{-1}\right)=-M(\sigma)^{-1} \mathfrak{x}(\sigma) .
\end{gathered}
$$

Soit a un vecteur de $\boldsymbol{R}^{k}$; posons

$$
\mathfrak{y}(\sigma)=\mathfrak{a}-M(\sigma) \mathfrak{a}
$$


pour tout $\sigma \in G$. Il est facile de voir que $\mathfrak{y}$ satisfait aux conditions (C1-2). Un tel cocycle $\mathfrak{y}$ s'appellera un cobord par rapport à $M$. On désigne par $B(M, \boldsymbol{R})$ l'espace linéaire des cobords par rapport à $M$; et l'on pose

$$
H(M, \boldsymbol{R})=Z(M, \boldsymbol{R}) / B(M, \boldsymbol{R}) .
$$

On appelle ce groupe quotient le groupe de cohomologie de $G$ par rapport à $M$.

Supposons maintenant que les coefficients de $M(\sigma)$ soient entiers pour tout $\sigma \in G$. Un cocycle parabolique x par rapport à $M$ sera dit entier si les composantes du vecteur $\mathfrak{x}(\sigma)$ sont entières pour tout $\sigma \in G$. On désigne par $Z(M, Z)$ (resp. $B(M, \boldsymbol{Z})$ ) le module des cocycles paraboliques (resp. cobords) entiers par rapport à $M$; et on pose

$$
H(M, \boldsymbol{Z})=Z(M, \boldsymbol{Z}) / B(M, \boldsymbol{Z}) .
$$

On voit que $H(M, \boldsymbol{Z})$ est canoniquement isomorphe à un sous-module de $H(M, \boldsymbol{R})$; on considère donc désormais $H(M, \boldsymbol{Z})$ comme un sous-module de $H(M, \boldsymbol{R})$.

Proposition 1. Toute base de $H(M, \boldsymbol{Z})$ sur $\boldsymbol{Z}$ donne une base de $H(M, \boldsymbol{R})$ sur $\boldsymbol{R}$.

On sait que $G$ a un système de générateurs en nombre fini $\left\{\sigma_{1}, \cdots, \sigma_{d}\right\}$. Si $\mathfrak{x}$ est un cocycle parabolique, on voit, en vertu de la condition (C1), que les vecteurs $\mathfrak{x}(\sigma)$ pour $\sigma \in G$ sont déterminés par les $d$ vecteurs $\mathfrak{x}\left(\sigma_{\nu}\right)$. On peut donc considérer $\mathfrak{x}$ comme un élément de $\boldsymbol{R}^{d k}=\boldsymbol{R}^{k} \times \cdots \times \boldsymbol{R}^{k}$ en identifiant $\mathfrak{x}$ avec le vecteur $\left(\mathfrak{x}\left(\sigma_{1}\right), \cdots, \mathfrak{x}\left(\sigma_{d}\right)\right) \in \boldsymbol{R}^{k} \times \cdots \times \boldsymbol{R}^{k} ; \mathfrak{x}$ appartient à $Z(M, \boldsymbol{Z})$ pourvu que les composantes du vecteur $\left(\mathfrak{x}\left(\sigma_{1}\right), \cdots, \mathfrak{x}\left(\sigma_{d}\right)\right)$ soient entières. On voit facilement que les conditions (C1-2) s'expriment sous la forme

$$
\sum_{\nu=1}^{d} L_{\mu \nu} \mathfrak{x}\left(\sigma_{\nu}\right)=0 \quad(\mu=1,2, \cdots)
$$

où les $L_{\mu \nu}$ sont des matrices de degré $k$ à coefficients entiers. Par conséquent, $Z(M, \boldsymbol{R})$ correspond à un sous-espace $V$ linéaire de $\boldsymbol{R}^{d k}$ défini sur le corps $\boldsymbol{Q}$ des nombres rationnels; et les éléments de $Z(M, Z)$ correspondent aux vecteurs de $V$ dont les composantes sont entières; il en est de même pour $B(M, \boldsymbol{R})$ et $B(M, \boldsymbol{Z})$. On peut en déduire l'assertion de la proposition.

Supposons que $Y$ ne soit pas vide et fixons un élément $\tau_{0}$ de $Y$. On désigne par $Z\left(M, \boldsymbol{R}, \tau_{0}\right)$ (resp. $\left.B\left(M, \boldsymbol{R}, \tau_{0}\right), Z\left(M, \boldsymbol{Z}, \tau_{0}\right), B\left(M, \boldsymbol{Z}, \tau_{0}\right)\right)$ l'ensemble des éléments $\mathfrak{x}$ de $Z(M, \boldsymbol{R})$ (resp. $B(M, \boldsymbol{R}), Z(M, \boldsymbol{Z}), B(M, \boldsymbol{Z})$ ) tels que $\mathfrak{x}\left(\tau_{0}\right)=0$; et l'on pose

$$
\begin{aligned}
& H\left(M, \boldsymbol{R}, \tau_{0}\right)=Z\left(M, \boldsymbol{R}, \tau_{0}\right) / B\left(M, \boldsymbol{R}, \tau_{0}\right), \\
& H\left(M, \boldsymbol{Z}, \tau_{0}\right)=Z\left(M, \boldsymbol{Z}, \tau_{0}\right) / B\left(M, \boldsymbol{Z}, \tau_{0}\right) .
\end{aligned}
$$

$H\left(M, \boldsymbol{Z}, \tau_{0}\right)$ s'identifie canoniquement avec un sous-module de $H\left(M, \boldsymbol{R}, \tau_{0}\right)$. On peut alors démontrer, de même que plus haut, que toute base de $H\left(M, \boldsymbol{Z}, \tau_{0}\right)$ 
sur $\boldsymbol{Z}$ forme une base de $H\left(M, \boldsymbol{R}, \tau_{0}\right)$ sur $\boldsymbol{R}$. Soit $\mathfrak{x}$ un élément de $Z(M, \boldsymbol{R})$. D'après la condition (C2), il existe un vecteur $\mathfrak{a} \in \boldsymbol{R}^{k}$ tel que

$$
\mathfrak{x}\left(\tau_{0}\right)=\mathfrak{a}-M\left(\tau_{0}\right) \mathfrak{a} .
$$

Posons $\mathfrak{y}(\sigma)=\mathfrak{a}-M(\sigma) \mathfrak{a}$ pour tout $\sigma \in G$; on voit alors que $\mathfrak{x}-\mathfrak{y}$ est contenu dans $Z\left(M, \boldsymbol{R}, \tau_{0}\right)$; on en conclut

$$
Z(M, \boldsymbol{R})=B(M, \boldsymbol{R})+Z\left(M, \boldsymbol{R}, \tau_{0}\right) .
$$

Il s'ensuit de là que $H\left(M, \boldsymbol{R}, \tau_{0}\right)$ est canoniquement isomorphe à $H(M, \boldsymbol{R})$. On constate de même que $H\left(M, \boldsymbol{Z}, \tau_{0}\right)$ est canoniquement isomorphe à un sousmodule $H^{\prime}$ de $H(M, \boldsymbol{Z})$ d'indice fini.

\section{§ 4. Périodes des intégrales.}

$G$ et $M$ étant comme dans le $\S 3$, supposons qu'il existe une matrice réelle $P$ de degré $k$ telle que l'on ait

$$
{ }^{t} M(\sigma) P M(\sigma)=P
$$

pour tout $\sigma \in G$. Désignons par $F(M)$ l'espace linéaire des formes différentielles vectorielles

$$
\omega=\left(\begin{array}{c}
\omega_{1} \\
\vdots \\
\omega_{k}
\end{array}\right)
$$

sur $\mathscr{H}$ jouissant des propriétés (D1-3) suivantes:

(D1) Chaque composante $\omega_{\nu}$ de $\omega$ est une forme différentielle fermêe de degré 1 , analytique par rapport à $x, y$, et à valeurs réelles.

(D2) On $a \omega \circ \sigma=M(\sigma) \omega$ pour tout $\sigma \in G$.

La condition (D1) entraîne que l'intégrale

$$
\int_{z}^{z^{\prime}} \omega
$$

ne dépend que de $z, z^{\prime}$ et non du choix du chemin d'intégration.

(D3) Pour chaque point parabolique s de G, la valeur limite

$$
\lim _{z \rightarrow s} \int_{z_{0}}^{z} \omega
$$

lorsque $z$ tend vers $s$, en restant dans un domaine fondamental de $G$, est déterminée et finie, $z_{0}$ étant un point què̀conque de $\mathscr{H}$.

Soit $\omega$ un élément de $F(M)$; fixons un point $z_{0}$ de $\mathscr{H}$ et posons

$$
\mathfrak{f}(z)=\int_{z_{0}}^{z} \omega+\mathfrak{a},
$$

où a est une «constante d'intégration» qui est un vecteur quelconque de $\boldsymbol{R}^{\boldsymbol{k}}$. Alors $\mathfrak{f}(z)$ est une fonction sur $\mathscr{H}$ à valeurs dans $\boldsymbol{R}^{k}$. En vertu de la propriété 
(D2), on obtient, pour chaque $\sigma \in G$,

$$
\mathfrak{f}(\sigma(z))=M(\sigma) \mathfrak{f}(z)+\mathfrak{x}(\sigma),
$$

où $\mathfrak{x}(\sigma)$ est le vecteur de $\boldsymbol{R}^{k}$ donné par

$$
\mathfrak{x}(\sigma)=\int_{z_{0}}^{\sigma\left(z_{0}\right)} \omega+\mathfrak{a}-M(\sigma) \mathfrak{a} .
$$

On vérifie facilement que $\mathfrak{x}$ satisfait à la condition $(\mathrm{C} 1)$ de $\S 3$. Soit $\tau$ une transformation parabolique de $G$, et soit $s$ le point fixe de $\tau$. D'après la propriété (D3), $\lim _{z \rightarrow s} \mathfrak{f}(z)$ est déterminé et fini; nous poserons

$$
\mathfrak{f}(s)=\lim _{z \rightarrow s} \mathfrak{f}(z) .
$$

On a alors $\mathfrak{f}(s)=\mathfrak{f}(\tau(s))=M(\tau) \mathfrak{f}(s)+\mathfrak{x}(\tau)$, de sorte qu'on a

$$
\mathfrak{x}(\tau)=[1-M(\tau)] \mathfrak{f}(s) .
$$

Il en résulte que $\mathfrak{x}$ est un élément de $Z(M, \boldsymbol{R})$. Le cocycle parabolique $\mathfrak{x}$ s'appellera la période de l'intégrale (11). On voit facilement que la classe de cohomologie de $\mathfrak{x}$ ne dépend que de $\omega$ et non du choix de $z_{0}$ et $\mathfrak{a}$.

Soit $\eta$ un autre élément de $F(M) ; \mathfrak{b}$ étant une constante d'intégration, posons

$$
\mathfrak{g}(z)=\int_{z_{0}}^{z} \eta+\mathfrak{b}
$$

On obtient alors

$$
\mathfrak{g}(\sigma(z))=M(\sigma) \mathfrak{g}(z)+\mathfrak{y}(\sigma),
$$

où $\mathfrak{y}$ est un cocycle parabolique donné par une formule analogue à (13). Considérons maintenant la forme différentielle ${ }^{t} \omega P \eta$; on voit que ${ }^{t} \omega P \eta$ est invariant par $G$. Posons

$$
(\omega, \eta)=\int_{\mathscr{I}}^{t} \omega P \eta,
$$

où $\mathscr{F}$ est un domaine fondamental quelconque de $G$. On va maintenant exprimer la valeur $(\omega, \eta)$ par les périodes $\mathfrak{x}(\sigma), \mathfrak{y}(\sigma)$, On voit d'abord qu'on a

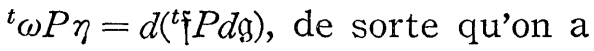

$$
(\omega, \eta)=\int_{\partial \mathscr{I}}{ }^{t} \mathfrak{F} P d \mathfrak{g},
$$

où $\partial \mathscr{I}$ désigne le bord de $\mathscr{I}$. On peut supposer que $\mathscr{I}$ est un polygone dont les côtés se correspondent deux à deux, en sens inverse, par des éléments de $G$; pour ainsi dire, on a

$$
\partial \mathscr{F}=\sum_{\mu}\left[E_{\mu}-\sigma_{\mu}\left(E_{\mu}\right)\right],
$$

où les $E_{\mu}$ sont des côtés qui forment la moitié du contour de $\mathscr{I}$ et les $\sigma_{\mu}$ 
sont des éléments de $G$. On a alors

$$
(\omega, \eta)=\sum_{\mu}\left(\int_{E_{\mu}}{ }^{t} \mathfrak{F} P d \mathfrak{g}-\int_{\sigma_{\mu}\left(E_{\mu}\right)}{ }^{t} \mathfrak{F} P d \mathfrak{g}\right) .
$$

Au moyen de (10), (12), (16), on obtient

$$
\begin{aligned}
\int_{\sigma_{\mu}\left(E_{\mu}\right)}{ }^{t} \mathfrak{f} P d \mathfrak{g} & =\int_{E_{\mu}}{ }^{t}\left(\mathfrak{f} \circ \sigma_{\mu}\right) P d\left(\mathfrak{g} \circ \sigma_{\mu}\right) \\
& =\int_{E_{\mu}}{ }^{t} \mathfrak{f} P d \mathfrak{g}+\int_{E_{\mu}}{ }^{t} \mathfrak{x}\left(\sigma_{\mu}\right) P M\left(\sigma_{\mu}\right) d \mathfrak{g} .
\end{aligned}
$$

En vertu des relations (9) et (10), on a enfin

$$
(\omega, \eta)=\sum_{\mu} t_{\mathfrak{c}}\left(\sigma_{\mu}^{-1}\right) P \int_{E_{\mu}} d \mathfrak{g} .
$$

Pour calculer plus explicitement, on adopte un domaine fondamental obtenu à partir d'une «dissection canonique» de la surface de Riemann comme suit. Soit $\mathscr{R}$ la surface de Riemann du corps des fonctions automorphes par rapport à $G$, et soit $g$ le genre de $R$. Soient $p_{1}, \cdots, p_{j}$ les points de $\mathcal{R}$ correspondant aux points elliptiques de $G$; et soient $q_{1}, \cdots, q_{h}$ les points de $\mathscr{R}$ correspondant aux points paraboliques de $G$. On prend un point $r_{0}$ de $\mathcal{R}$ autre que $p_{\mu}, q_{\nu}$; on coupe $R$ par $2 g$ courbes fermées passant $r_{0}$ qui forment un système de générateurs canoniques du groupe fondamental de $\mathscr{R}$, où l'on suppose que ces courbes ne traversent aucun des points $p_{\mu}, q_{\nu}$. On obtient alors un polygone à $4 g$ côtés contenant les $p_{\mu}, q_{\nu}$ dans son intérieur. On coupe en outre ce polygone le long de $j+h$ segments joignant un sommet au $p_{\mu}, q_{\nu}$; on obtient ainsi un polygone $G$ à $4 g+2 j+2 h$ côtés. Prenons pour domaine fondamental de $G$ un polygone $\mathscr{F}$ dans $\mathscr{H}$ correspondant au polygone $\mathcal{G}$, et désignons par $t_{\mu}, s_{\nu}$ les sommets de $\mathscr{I}$ correspondant aux $p_{\mu}, q_{\nu}$. D'après notre construction, le bord $\partial \mathscr{F}$ de $\mathscr{I}$ est la somme de $4 g+2 j+2 h$ côtés

$$
\begin{gathered}
A_{1}, B_{1},-\alpha_{1}\left(A_{1}\right),-\beta_{1}^{-1}\left(B_{1}\right), \cdots, A_{g}, B_{g},-\alpha_{g}\left(A_{g}\right),-\beta_{g}{ }^{-1}\left(B_{g}\right), \\
C_{1},-\gamma_{1}\left(C_{1}\right), \cdots, C_{j},-\gamma_{j}\left(C_{j}\right), \\
D_{1},-\delta_{1}\left(D_{1}\right), \cdots, D_{h},-\delta_{h}\left(D_{h}\right),
\end{gathered}
$$

où les $\alpha_{\lambda}, \beta_{\lambda}, \gamma_{\mu}, \delta_{\nu}$ sont des éléments de $G$ tels que

$$
\begin{array}{cr}
\delta_{h} \cdots \delta_{1} \gamma_{j} \cdots \gamma_{1} \beta_{g}{ }^{-1} \alpha_{g}{ }^{-1} \beta_{g} \alpha_{g} \cdots \beta_{1}{ }^{-1} \alpha_{1}{ }^{-1} \beta_{1} \alpha_{1} & =1, \\
\gamma_{\mu}{ }^{e \mu}=1 & (\mu=1, \cdots, j) ;
\end{array}
$$

$\gamma_{\mu}$ est une transformation elliptique qui laisse fixe $t_{\mu} ; e_{\mu}$ est l'ordre de ramification en $t_{\mu}$; et $\delta_{\mu}$ est une transformation parabolique qui laisse fixe le point parabolique $s_{\mu}$. Posons

$$
\sigma_{\lambda}=\beta_{\lambda}{ }^{-1} \alpha_{\lambda}{ }^{-1} \beta_{\lambda} \alpha_{\lambda}, \quad \tau_{\lambda}=\sigma_{\lambda} \cdots \sigma_{1} \quad(\lambda=1, \cdots, g) .
$$


Soit $u_{0}$ le point de départ de $A_{1}$; et posons

$$
\begin{array}{ll}
u_{\lambda} & =\tau_{\lambda}\left(u_{0}\right), \\
v_{0} & =\gamma_{j} \cdots r_{1}\left(u_{g}\right) .
\end{array} \quad(\lambda=1, \cdots, g),
$$

Alors, $A_{\lambda}, B_{\lambda}, C_{\mu}, D_{\nu}$ joignent respectivement $u_{\lambda-1}$ à $\alpha_{\lambda}^{-1} \beta_{\lambda} \alpha_{\lambda}\left(u_{\lambda-1}\right), \alpha_{\lambda}^{-1} \beta_{\lambda} \alpha_{\lambda}\left(u_{\lambda-1}\right)$ à $\beta_{\lambda} \alpha_{\lambda}\left(u_{\lambda-1}\right), \gamma_{\mu-1} \cdots \gamma_{1}\left(u_{g}\right)$ à $t_{\mu}, \delta_{\nu-1} \cdots \delta_{1}\left(v_{0}\right)$ à $s_{\nu}$. On a donc, au moyen de (17),

$$
\begin{aligned}
(\omega, \eta)= & \sum_{\lambda=1}^{g} t_{\mathfrak{x}}\left(\alpha_{\lambda}^{-1}\right) P\left[\mathfrak{g}\left(\alpha_{\lambda}^{-1} \beta_{\lambda} \alpha_{\lambda}\left(u_{\lambda-1}\right)\right)-\mathfrak{g}\left(u_{\lambda-1}\right)\right] \\
& +\sum_{\lambda=1}^{g} t_{\mathfrak{t}}\left(\beta_{\lambda}\right) P\left[\mathfrak{g}\left(\beta_{\lambda} \alpha_{\lambda}\left(u_{\lambda-1}\right)\right)-\mathfrak{g}\left(\alpha_{\lambda}^{-1} \beta_{\lambda} \alpha_{\lambda}\left(u_{\lambda-1}\right)\right)\right] \\
& +\sum_{\mu=1}^{j} t_{\mathfrak{t}} \mathfrak{x}\left(\gamma_{\mu}{ }^{-1}\right) P\left[\mathfrak{g}\left(t_{\mu}\right)-\mathfrak{g}\left(\gamma_{\mu-1} \cdots \gamma_{1}\left(u_{g}\right)\right)\right] \\
& +\sum_{\nu=1}^{h} t_{\mathfrak{t}} \mathfrak{x}\left(\delta_{\nu}{ }^{-1}\right) P\left[\mathfrak{g}\left(s_{\nu}\right)-\mathfrak{g}\left(\delta_{\nu-1} \cdots \delta_{1}\left(v_{0}\right)\right)\right] .
\end{aligned}
$$

Comme $\gamma_{\mu}$ laisse fixe le point $t_{\mu}$, on a

$$
\mathrm{g}\left(t_{\mu}\right)=\mathfrak{g}\left(r_{\mu}{ }^{m}\left(t_{\mu}\right)\right)=M\left(r_{\mu}{ }^{m}\right) \mathfrak{g}\left(t_{\mu}\right)+\mathfrak{y}\left(r_{\mu}{ }^{m}\right)
$$

pour $m=0,1, \cdots, e_{\mu}-1$; on en déduit

$$
e_{\mu} \mathrm{g}\left(t_{\mu}\right)=\sum_{m=1}^{e^{\mu-1}} M\left(\gamma_{\mu}{ }^{m}\right) \mathfrak{g}\left(t_{\mu}\right)+\sum_{m=1}^{e \mu^{-1}} \mathfrak{y}\left(\gamma_{\mu}{ }^{m}\right)
$$

D'autre part, on a

$$
0=\mathfrak{x}(1)=\mathfrak{x}\left(\gamma_{\mu}^{-e} \mu\right)=\sum_{m=1}^{e_{\mu}-1} M\left(\gamma_{\mu}^{-m}\right) \mathfrak{x}\left(\gamma_{\mu}^{-1}\right)
$$

de là et de (9), on obtient

$$
{ }^{t} \mathfrak{x}\left(\gamma_{\mu}{ }^{-1}\right) P \mathfrak{g}\left(t_{\mu}\right)={ }^{t} \mathfrak{x}\left(\gamma_{\mu}{ }^{-1}\right) P\left[e_{\mu}{ }^{-1} \sum_{m=1}^{\epsilon} \mu^{-1} \mathfrak{y}\left(\gamma_{\mu}{ }^{m}\right)\right] .
$$

Posons $\mathfrak{g}\left(u_{0}\right)=\mathfrak{r}$; on a alors $\mathfrak{g}\left(\rho\left(u_{0}\right)\right)=M(\rho) \mathfrak{r}+\mathfrak{y}(\rho)$ pour tout $\rho \in G$. Au moyen de cette relation et (18), on a

$$
\begin{aligned}
(\omega, \eta)= & \sum_{\lambda=1}^{g} t_{\mathfrak{x}}\left(\alpha_{\lambda}^{-1}\right) P\left[\mathfrak{y}\left(\alpha_{\lambda}^{-1} \beta_{\lambda} \alpha_{\lambda} \tau_{\lambda-1}\right)-\mathfrak{y}\left(\tau_{\lambda-1}\right)\right] \\
& +\sum_{\lambda=1}^{g} t_{\mathfrak{x}}\left(\beta_{\lambda}\right) P\left[\mathfrak{y}\left(\beta_{\lambda} \alpha_{\lambda} \tau_{\lambda-1}\right)-\mathfrak{y}\left(\alpha_{\lambda}^{-1} \beta_{\lambda} \alpha_{\lambda} \tau_{\lambda-1}\right)\right] \\
& +\sum_{\mu=1}^{j} t_{\mathfrak{x}}\left(\gamma_{\mu}^{-1}\right) P\left[e_{\mu}^{-1} \sum_{m=1}^{e_{\mu}-1} \mathfrak{y}\left(\gamma_{\mu}^{m}\right)-\mathfrak{y}\left(\gamma_{\mu-1} \cdots \gamma_{1} \tau_{g}\right)\right] \\
& -\sum_{\nu=1}^{n} t^{t} \mathfrak{x}\left(\delta_{\nu}{ }^{-1}\right) P \mathfrak{y}\left(\delta_{\nu-1} \cdots \delta_{1} \gamma_{j} \cdots \gamma_{1} \tau_{g}\right)+\sum_{\nu=1}^{n} t_{\mathfrak{x}}\left(\delta_{\nu}{ }^{-1}\right) P \mathfrak{g}\left(s_{\nu}\right)+\psi(\mathfrak{l}),
\end{aligned}
$$


où $\psi(\mathfrak{r})$ est le nombre donné par

$$
\begin{aligned}
\psi(\mathfrak{r})= & \sum_{\lambda=1}^{g} t_{\mathfrak{x}}\left(\alpha_{\lambda}^{-1}\right) P\left[M\left(\alpha_{\lambda}^{-1} \beta_{\lambda} \alpha_{\lambda} \tau_{\lambda-1}\right)-M\left(\tau_{\lambda-1}\right)\right] \mathfrak{r} \\
& +\sum_{\lambda=1}^{g} t_{\mathfrak{x}}\left(\beta_{\lambda}\right) P\left[M\left(\beta_{\lambda} \alpha_{\lambda} \tau_{\lambda-1}\right)-M\left(\alpha_{\lambda}{ }^{-1} \beta_{\lambda} \alpha_{\lambda} \tau_{\lambda-1}\right)\right] \mathfrak{r} \\
& -\sum_{\mu=1}^{j} t_{\mathfrak{x}}\left(\gamma_{\mu}^{-1}\right) P M\left(\gamma_{\mu-1} \cdots \gamma_{1} \tau_{g}\right) \mathfrak{r} \\
& -\sum_{\nu=1}^{n} t_{\mathfrak{x}}\left(\delta_{\nu}{ }^{-1}\right) P M\left(\delta_{\nu-1} \cdots \delta_{1} \gamma_{j} \cdots \gamma_{1} \tau_{g}\right) \mathfrak{r} .
\end{aligned}
$$

On va maintenant démontrer $\psi(\mathfrak{r})=0$. D'après la relation $(10)$, on a

$$
\psi(\mathfrak{r})={ }^{t} \psi(\mathfrak{r})={ }^{t} \mathfrak{r}^{t} P\left(\sum_{\lambda=1}^{g} \mathfrak{c}_{\lambda}-\sum_{\mu=1}^{j} \mathfrak{d}_{\mu}-\sum_{\nu=1}^{h} \mathfrak{e}_{\nu}\right),
$$

où $c_{\lambda}, \grave{\partial}_{\mu}, \mathfrak{e}_{\nu}$ sont les vecteurs donnés par

$$
\begin{aligned}
\mathfrak{c}_{\lambda}= & M\left(\tau_{\lambda-1}{ }^{-1}\right)\left[M\left(\alpha_{\lambda}{ }^{-1} \beta_{\lambda}^{-1} \alpha_{\lambda}\right) \mathfrak{r}\left(\alpha_{\lambda}^{-1}\right)-\mathfrak{r}\left(\alpha_{\lambda}{ }^{-1}\right)\right. \\
& \left.\quad+M\left(\alpha_{\lambda}^{-1} \beta_{\lambda}{ }^{-1}\right) \mathfrak{r}\left(\beta_{\lambda}\right)-M\left(\alpha_{\lambda}{ }^{-1} \beta_{\lambda}^{-1} \alpha_{\lambda}\right) \mathfrak{r}\left(\beta_{\lambda}\right)\right], \\
\mathfrak{\searrow}_{\mu}= & M\left(\tau_{g}{ }^{-1} \gamma_{1}{ }^{-1} \cdots \gamma_{\mu-1}{ }^{-1}\right) \mathfrak{r}\left(\gamma_{\mu}{ }^{-1}\right), \\
\mathfrak{e}_{\nu}= & M\left(\tau_{g}{ }^{-1} \gamma_{1}{ }^{-1} \cdots \gamma_{j}^{-1} \delta_{1}^{-1} \cdots \delta_{\nu-1}{ }^{-1}\right) \mathfrak{r}\left(\delta_{\nu}{ }^{-1}\right) .
\end{aligned}
$$

On voit facilement

$$
\begin{aligned}
& \mathfrak{c}_{\lambda}=-M\left(\tau_{\lambda-1}{ }^{-1}\right) \mathfrak{r}\left(\alpha_{\lambda}{ }^{-1} \beta_{\lambda}{ }^{-1} \alpha_{\lambda} \beta_{\lambda}\right)=\mathfrak{x}\left(\tau_{\lambda-1}{ }^{-1}\right)-\mathfrak{x}\left(\tau_{\lambda}{ }^{-1}\right), \\
& -\mathfrak{b}_{\mu}=\mathfrak{x}\left(\tau_{g}{ }^{-1} \gamma_{1}{ }^{-1} \cdots \gamma_{\mu-1}{ }^{-1}\right)-\mathfrak{x}\left(\tau_{g}{ }^{-1} \gamma_{1}{ }^{-1} \cdots \gamma_{\mu}{ }^{-1}\right) \text {, } \\
& -\mathfrak{e}_{\nu}=\mathfrak{x}\left(\tau_{g}{ }^{-1} \gamma_{1}{ }^{-1} \cdots \gamma_{j}{ }^{-1} \delta_{1}^{-1} \cdots \delta_{\nu-1}{ }^{-1}\right)-\mathfrak{x}\left(\tau_{g}{ }^{-1} \gamma_{1}{ }^{-1} \cdots \gamma_{j}{ }^{-1} \delta_{1}{ }^{-1} \cdots \delta_{\nu}{ }^{-1}\right) \text {. }
\end{aligned}
$$

D'après la relation $\tau_{g}{ }^{-1} \gamma_{1}{ }^{-1} \cdots \gamma_{j}{ }^{-1} \delta_{1}^{-1} \cdots \delta_{h}{ }^{-1}=1$, on obtient

$$
\psi(\mathfrak{r})=0 \text {. }
$$

\section{$\S 5$. L'isomorphisme de $S_{m}(G)$ sur le groupe de cohomologie.}

Soit $n$ un entier pair non-négatif; soit $U$ une matrice inversible de degré $n+1$ à coefficients réels; posons

$$
P={ }^{t} U^{-1} P_{n} U^{-1}, \quad M(\sigma)=U M_{n}(\sigma) U^{-1}
$$

pour $\sigma \in \mathrm{GL}(2, \boldsymbol{R}), M_{n}$ étant la représentation définie au $\S 1 ; M$ est une représentation réelle de $G L(2, \boldsymbol{R})$, et on a

$$
{ }^{t} M(\sigma) P M(\sigma)=P
$$

pour tout $\sigma \in \mathrm{SL}(2, \boldsymbol{R})$. Soit $f$ un élément de $S_{n+2}(G)$; considérons la forme différentielle vectorielle $\operatorname{Re}\left(U f d_{z_{n}}\right)$ sur $\mathscr{K}$, où $d_{z_{n}}$ est la forme dèfinie au $\S 2$. 
Comme $f d_{z_{n}}$ est holomorphe sur $\mathscr{H}$, on a $d\left(\operatorname{Re}\left(U f d_{z_{n}}\right)\right)=0$; en vertu de (5), on a

$$
\operatorname{Re}\left(U f d_{z_{n}}\right) \circ \sigma=M(\sigma) \operatorname{Re}\left(U f d_{\partial_{n}}\right)
$$

pour tout $\sigma \in G$; la propriété (P3) de la forme parabolique $f$ entraîne la condition (D3) pour $\operatorname{Re}\left(U f d_{z_{n}}\right)$. Il en résulte que $\operatorname{Re}\left(U f d_{z_{n}}\right)$ est un élément de $F(M)$. On a vu au $\S 4$ que la période de l'intégrale d'un élément de $F(M)$ définit un élément de $H(M, \boldsymbol{R})$. Désignons par $\varphi(f)$ l'élément de $H(M, \boldsymbol{R})$ correspondant à la forme $\operatorname{Re}\left(U f d_{b_{n}}\right) ; \varphi$ est alors une application $\boldsymbol{R}$-linéaire de $S_{n+2}(G)$ dans $H(M, \boldsymbol{R})$.

THÉORÈme 1. Les notations étant comme ci-dessus, $\varphi$ est un isomorphisme de $S_{n+2}(G)$ sur $H(M, \boldsymbol{R})$.

D'après un résultat d'Eichler [1], les espaces $S_{n+2}(G)$ et $H(M, \boldsymbol{R})$ sont de la même dimension sur $\boldsymbol{R}$. Il nous faut donc seulement démontrer que le noyau de $\varphi$ est $\{0\}$. Soient $f$, $g$ deux éléments de $S_{n+2}(G)$; posons $\omega=\operatorname{Re}\left(U f d_{\jmath_{n}}\right)$, $\eta=\operatorname{Re}\left(U g d_{\partial_{n}}\right)$. On a alors, d'après la formule (8) de $\S 2$,

$$
\Lambda(f, g)=(-1)^{\frac{n}{2}+1}(\omega, \eta) .
$$

La valeur $\Lambda(f, g)$ est donc donnée par (19). Supposons qu'on ait $\varphi(f)=0$. Alors on peut choisir la constante a de l'intégrale (11) de telle manière que le cocycle parabolique $\mathfrak{x}$ déterminé par (13) soit 0 . D'après (19)] et [20), on a $\Lambda(f, g)=0$ pour tout $g \in S_{n+2}(G)$; en particulier, on a $\Lambda(f$, if $)=0$, de sorte qu'on a $f=0$; ce qui complète la démonstration.

\section{§ 6. Les variétés abéliennes attachées aux formes paraboliques.}

Considérons maintenant le cas où la représentation $M_{n}$ de $G$ satisfait à la condition suivante.

(A) Il existe une matrice inversible $U$ de degré $n+1$ à coefficients réels telle que ${ }^{t} U^{-1} P_{n} U^{-1}$ et $U M_{n}(\sigma) U^{-1}$ pour tout $\sigma \in G$ soient des matrices à coefficients entiers.

Posons

$$
P={ }^{t} U^{-1} P_{n} U^{-1}, \quad M(\sigma)=U M_{n}(\sigma) U^{-1}
$$

pour tout $\sigma \in \mathrm{GL}(2, \boldsymbol{R})$. Soit $\varphi$ l'isomorphisme de $S_{n+2}(G)$ sur $H(M, \boldsymbol{R}) \mathrm{du}$ théorème 1 ; posons

$$
D_{n+2}(G)=\varphi^{-1}[H(M, Z)] .
$$

On voit alors, d'après la proposition 1 , que $D_{n+2}(G)$ est un lattice dans l'espace vectoriel $S_{n+2}(G)$, de sorte que l'espace quotient $S_{n+2}(G) / D_{n+2}(G)$ est un tore complexe. Nous allons maintenant démontrer notre théorème principal.

THÉORÈmE 2. Les notations et les hypothèses étant comme ci-dessus, il existe 
un entier positif $c$ tel que la forme $c(f, g)$ donne une forme de Riemann sur $S_{n+2}(G) / D_{n+2}(G)$.

Il en résulte que $S_{n+2}(G) / D_{n+2}(G)$ a une structure de variété abélienne.

On entend ici par une forme de Riemann sur le tore complexe $S_{m}(G) / D_{m}(G)$ une forme hermitienne positive sur $S_{m}(G)$ dont la partie imaginaire est à valeurs entières sur $D_{m}(G) \times D_{m}(G)$; on s'en réfère à Weil [7].

Le théorème sera démontré si nous faisons voir que la valeur $\Lambda(f, g)$ est rationnelle pour tout $f, g \in D_{n+2}(G)$. Pour cela, on se sert de la formule (19). Soient $f, g$ deux éléments de $D_{n+2}(G)$. Posons $\omega=\operatorname{Re}\left(U f d_{\hat{z}_{n}}\right), \eta=\operatorname{Re}\left(U g d_{z_{n}}\right)$, et définissons pour $\omega, \eta$ les fonctions $\mathfrak{f}, \mathfrak{g}$ et les cocycles paraboliques $\mathfrak{x}, \mathfrak{y}$ comme dans le $\S 4$. D'après la définition de $D_{n+2}(G)$, on peut prendre les vecteurs $\mathfrak{a}, \mathfrak{b}$ dans (11), (15) de telle façon que $\mathfrak{x}(\sigma), \mathfrak{y}(\sigma)$ soient des vecteurs à composantes entières pour tout $\sigma \in G$. $\mathfrak{x}, \mathfrak{y}$ étant définis ainsi, la valeur $\Lambda(f, g)$ est donnée par (19)] et (20). Il est clair que les quatre premières sommes de (19)] sont des nombres rationnelles. Comme les $\delta_{\nu}$ sont des transformations paraboliques, il existe, pour chaque $\nu$, un vecteur $\mathfrak{b}_{\nu}$ tel que l'on ait

$$
\mathfrak{x}\left(\delta_{\nu}{ }^{-1}\right)=\left[1-M\left(\delta_{\nu}{ }^{-1}\right)\right] \mathfrak{b}_{\nu} .
$$

Comme les composantes du vecteur $\mathfrak{r}\left(\delta_{\nu}{ }^{-1}\right)$ sont entières, on peut supposer que les composantes de $\mathfrak{b}_{\nu}$ sont des nombres rationnels. On voit alors que la cinquième somme de (19)] est égale à

$$
\begin{aligned}
\sum_{\nu=1}^{h} t_{\mathfrak{y}_{\nu}}{ }^{t}\left(1-M\left(\delta_{\nu}{ }^{-1}\right)\right) P \mathfrak{g}\left(s_{\nu}\right) & =\sum_{\nu=1}^{h} t_{\mathfrak{b}_{\nu}} P\left(1-M\left(\delta_{\nu}\right)\right) \mathfrak{g}\left(s_{\nu}\right) \\
& =\sum_{\nu=1}^{h} t_{\mathfrak{y}_{\nu}} P \mathfrak{y}\left(\delta_{\nu}\right) ;
\end{aligned}
$$

ce nombre est évidemment rationnel. Quant au dernier terme $\psi(\mathfrak{r})$ de (19), on a déjà vu qu'il est égal à 0 . Il en résulte que la valeur $\Lambda(f, g)$ est rationnelle pour $f, g \in D_{n+2}(G)$; ceci achève la démonstration.

On voit que, si $n=0$, la variété abélienne $S_{2}(G) / D_{2}(G)$ n'est autre que la variété jacobienne du corps des fonctions automorphes par rapport à $G$.

Soit $G^{\prime}$ un sous-groupe de $G$ d'indice fini; on observe que $S_{m}(G)$ est contenu dans $S_{m}\left(G^{\prime}\right)$ et $D_{m}(G)$. est contenu dans $D_{m}\left(G^{\prime}\right)$. Par suite il existe une isogénie de la variété abélienne $S_{m}(G) / D_{m}(G)$ sur une sous-variété abélienne de $S_{m}\left(G^{\prime}\right) / D_{m}\left(G^{\prime}\right)$.

\section{§ 7. L'algèbre des transformations.}

Soit $\&$ un groupe quelconque; deux sous-groupes $G, G^{\prime}$ de $\mathbb{B}$ sont dits commensurables si l'intersection $G \cap G^{\prime}$ est d'indice fini dans $G$ et dans $G^{\prime}$. Fixons un sous-groupe $G$ de $\mathbb{S}$; soit $\tilde{G}$ l'ensemble de tous les éléments $\rho$ de (B) tels que $\rho^{-1} G \rho$ soit commensurable avec $G$. Il est facile de voir que $\tilde{G}$ 


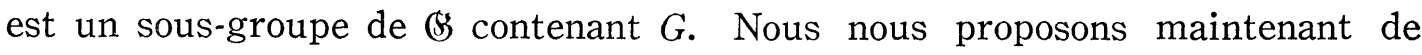
construire, d'après une idée de $\mathrm{A}$. Weil, une algèbre $\mathfrak{A}$ à partir des éléments de $\tilde{G}$. Soit $\mathfrak{M}$ l'ensemble des classes doubles $G \rho G$ pour' $\rho \in \tilde{G}$, et soit $\mathfrak{A}$ le $\boldsymbol{Z}$-module libre engendré par les éléments de $\mathfrak{M}$. On va définir une loi de multiplication sur $\mathfrak{A}$. On observe d'abord que, pour tout $\rho \in G$, le nombre des classes à droite $G \alpha$ contenues dans $G \rho G$ est fini et égal à l'indice $\left[G: G \cap \rho^{-1} G \rho\right]$; en effet, comme $\rho^{-1} G \rho$ est commensurable avec $G$, il existe un nombre fini d'éléments $\left\{\gamma_{\nu}\right\}$ tel que

On a alors

$$
G=\bigcup_{\nu}\left(G \cap \rho^{-1} G \rho\right) \gamma_{\nu} .
$$

$$
G \rho G=\bigcup_{\nu} G \rho \gamma_{\nu} .
$$

Il est facile de voir que les $G \rho \gamma_{\nu}$ sont distincts si les $\left(G \cap \rho^{-1} G \rho\right) \gamma_{\nu}$ sont distincts.

Lemme 1. Soient $A, B=G \psi G, C$ trois éléments de $\mathfrak{M}$ et $G \omega$ une classe à droite suivant $G$ contenue dans $C$. Soit $\left\{\psi_{1} G, \cdots, \psi_{s} G\right\}$ le système complet des classes à gauche suivant $G$ contenues dans $G \psi^{-1} G$. Alors, le nombre de $\nu$ tel que l'on ait $G \omega \psi_{\nu} G=A$ ne dépend que de $A, B, C$ et non du choix de $\omega,\left\{\psi_{\nu}\right\}$.

Il est clair que le nombre en question ne dépend pas du choix de $\left\{\psi_{\nu}\right\}$. Considérons donc une autre classe à droite $G \omega^{\prime}$ contenue dans $C$. Alors, il existe un élément $\gamma \in G$ tel que $G \omega=G \omega^{\prime} \gamma$. On voit que l'ensemble $\left\{\gamma \psi_{1} G, \cdots, \gamma \psi_{s} G\right\}$ coïncide, pris dans sa totalité, avec $\left\{\psi_{1} G, \cdots, \psi_{s} G\right\}$, et on a $\left(G \omega^{\prime}\right)\left(\gamma \psi_{\nu} G\right)=G \omega \psi_{\nu} G$, d'où résulte l'assertion du lemme.

On désignera par $(A \cdot B, C)$ le nombre du lemme 1 .

Lemme 2. Soient $A=G \rho G, B=G \psi G$ deux éléments de $\mathfrak{M}$; soient $\left\{G \rho_{1}, \cdots\right.$, $\left.G \rho_{r}\right\}$ et $\left\{G \psi_{1}, \cdots, G \psi_{s}\right\}$ respectivement les systèmes complets des classes à droite suivant $G$ contenues dans $A$ et $B$. Alors, le nombre de $(\mu, \nu)$ tel que $G \rho_{\mu} \psi_{\nu}=G \rho \psi$ est égal à $(A \cdot B, G \rho \psi G)$.

Supposons qu'on ait $G \rho_{\mu} \psi_{\nu}=G \rho \psi$; on a alors $G \rho \psi \psi_{\nu}^{-1}=G \rho_{\mu}$ et donc $G \rho \psi \psi_{\nu}{ }^{-1} G=G \rho_{\mu} G=G \rho G$. Réciproquement, si l'on a $G \rho \psi \psi_{\nu}{ }^{-1} G=G \rho G$, la classe à droite $G \rho \psi \psi_{\nu}{ }^{-1}$ coïncide avec $G \rho_{\mu}$ pour un et un seul $\mu$, de sorte qu'alors on a $G \rho \psi=G \rho_{\mu} \psi_{\nu}$. Par suite, le nombre de $(\mu, \nu)$ tel que $G \rho_{\mu} \psi_{\nu}$ $=G \rho \psi$ est égal au nombre de $\nu$ tel que

$$
(G \rho \psi) \psi_{\nu}{ }^{-1} G=G \circ G,
$$

c'est à dire $(A \cdot B, G \rho \psi G)$, compte tenu du fait que $\left\{\psi_{1}^{-1} G, \cdots, \psi_{s}^{-1} G\right\}$ donne le système complet des classes à gauche suivant $G$ contenues dans $G \psi^{-1} G$.

Les notations étant comme dans le lemme 2, on a

$$
G \rho G \psi G=\bigcup_{\mu, \nu} G \rho_{\mu} \psi_{\nu} .
$$


D'après le lemme 2 , la classe $G \rho_{\alpha} \psi_{\beta}$ figure justement $\left(A \cdot B, G \rho_{\alpha} \psi_{\beta} G\right)$ fois parmi $\left\{G \rho_{\mu} \psi_{\nu}\right\}$. Or on définit le produit $A \cdot B$ par

$$
A \cdot B=\Sigma(A \cdot B, C) C,
$$

où la somme est étendue à toutes les classes $C=G \tau G$ contenues dans $G \rho G \psi G$. En prolongeant par linéarité cette opération sur $\mathfrak{A} \times \mathfrak{A}$, on obtient une structure d'anneau sur $\mathfrak{A}$. Pour démontrer l'associativité, considérons un espace (5) sur lequel $\tilde{G}$ opère à gauche. Désignons par $\mathfrak{D}=\mathfrak{D}(\mathbb{E} / G)$ le $\boldsymbol{Z}$-module libre engendré par les éléments de $\leftleftarrows / G$. Chaque élément $A=G \rho G$ de $\mathfrak{M}$ donne alors un endomorphisme de $\mathfrak{D}$ comme suit. Notons $\pi$ l'application canonique de $(5)$ sur $(\xi / G$. Soit $p$ un point de $\& / G$ et soit $\xi$ un point de $\&$ tel que $\pi(\xi)=p . \quad\left\{\rho_{\mu}\right\}$ étant comme plus haut, posons

$$
A(p)=\sum_{\mu} \pi\left(\rho_{\mu} \xi\right) \text {. }
$$

On voit aisément que cette définition ne dépend pas du choix de $\left\{\rho_{\mu}\right\}$. On prolonge, par linéarité, l'opération sur $\mathfrak{X} \times \mathfrak{D}$. D'après notre définition et d'après le lemme 2 , on a $A \cdot B(p)=A(B(p))$ pour tout $A, B \in \mathfrak{M}$ et tout $p \in[/ G$. On obtient ainsi une représentation de l'anneau $\mathfrak{A}$ par des endomorphismes du module $\mathfrak{D}$. Prenons en particulier $\tilde{G}$ pour $\mathfrak{F}$. On vérifie alors facilement que la représentation est fidèle, ce qui démontre l'associativité de l'anneau $\mathfrak{A}$. On appelle $\mathfrak{A}$ l'anneau des transformations de $G$ par rapport à $\mathbb{G}$.

Considérons le cas où $G$ est un groupe fuchsien sur $\mathscr{H}$ et $\mathscr{G}=\operatorname{SL}(2, \boldsymbol{R}) /$ $\left\{ \pm I_{2}\right\}$. Soit $\mathscr{H}^{*}$ la réunion de $\mathscr{H}$ et de l'ensemble des points paraboliques pour $G$. On observe alors que $\tilde{G}$ opère sur $\mathscr{H}^{*}$. Soit $\mathbb{6}$ une courbe algébrique dont le corps des fonctions est le corps des fonctions automorphes par rapport à $G$. Alors, l'espace quotient $\mathscr{H}^{*} / G$ s'identifie avec $\mathbb{S}$ et le module $\mathfrak{D}\left(\mathscr{S}^{*} / G\right)$ avec le module des diviseurs de la courbe 5 . Soit $\pi$ l'application canonique de $\mathscr{I}^{*}$ sur $\mathfrak{E}$. $\mathfrak{M}, \mathfrak{A}$ étant définis pour $G$ comme plus haut, soit $A=G \rho G$ un élément de $\mathfrak{M}$. On vérifie facilement que l'ensemble des points de $\mathbb{5} \times \mathbb{S}$ de la forme $\pi(z) \times \pi(\rho(z))$ pour $z \in \mathcal{H}^{*}$ est une sous-variété $X$ de $\mathfrak{E} \times \mathbb{(}$, de dimension 1 ; $X$ ne dépend que de $A$ et non du choix de $\rho$. On peut démontrer sans peine que l'application $A \rightarrow X$ donne un isomorphisme de l'anneau $\mathfrak{A}$ dans l'anneau des correspondances algébriques de la courbe $\mathfrak{5}$. L'opération de $A$ sur $\mathfrak{D}\left(\mathscr{H}^{*} / G\right)$ donnée plus haut coïncide avec l'opération de $X$ définie dans Weil [8], On voit de plus que la transposée $X^{\prime}$ de $X$ correspond à la classe $G \rho^{-1} G$.

\section{§ 8. Les opérateurs de Hecke.}

Soit $G$ un sous-groupe discret de $\operatorname{SL}(2, \boldsymbol{R})$ tel que $\mathscr{H} / G$ ait une mesure finie. Nous allons maintenant définir des représentations de l'anneau des 
transformations de $G$ dans $S_{m}(G)$.

Soit $\rho$ un élément de $\mathrm{GL}_{+}(2, \boldsymbol{R})$ tel que $\rho^{-1} G \rho$ soit commensurable avec $G$. Comme on a vu au $\S 7$, il existe un système $\left\{\rho_{\mu}\right\}$ des éléments en nombre fini tel que l'on ait

$$
G \rho G=\bigcup_{\mu} G \rho_{\mu}
$$

et que les $G \rho_{\mu}$ soient distincts. Soit $f$ un élément de $S_{m}(G)$; on définit $g=f$. I par

$$
g(z)=(\operatorname{det} \rho)^{m-1} \sum_{\mu} f\left(\rho_{\mu}(z)\right) J\left(\rho_{\mu}, z\right)^{m} .
$$

On constate que $g$ est un élément de $S_{m}(G)$ et que $\mathfrak{I}$ ne dépend que de la classe $G \rho G$. On posera $\mathfrak{I}=\mathfrak{I}(\rho)=\mathfrak{I}(G \rho G)$. On a, pour tout $\rho \in \mathrm{GL}_{+}(2, \boldsymbol{R})$ et pour tout nombre positif $a$,

$$
\mathfrak{I}(\alpha \rho)=a^{m-2 \mathfrak{I}}(\rho) .
$$

Soit $\mathfrak{A}$ l'anneau des transformations de $G$ par rapport à $\mathrm{SL}(2, \boldsymbol{R})$. Alors, on déduit de notre définition de $\mathfrak{A}$ et de $\mathfrak{I}$, que l'application $G \rho G \rightarrow \mathfrak{I}(G \rho G)$ donne une représentation de $\mathfrak{A}$ dans $S_{m}(G)$.

Proposition 2. Soit $\rho$ un élément de $\mathrm{GL}_{+}(2, \boldsymbol{R})$; posons

$$
\rho^{*}=(\operatorname{det} \rho) \rho^{-1} \text {. }
$$

Alors, on a, pour tout $f, g \in S_{m}(G)$,

$$
(f \cdot \mathfrak{I}(\rho), g)=\left(f, g \cdot \mathfrak{I}\left(\rho^{*}\right)\right) \text {. }
$$

Posons $m=n+2, \mathfrak{I}=\mathfrak{T}(\rho), \mathfrak{T}^{*}=\mathfrak{I}\left(\rho^{*}\right)$ et $q=\operatorname{det} \rho$. On a alors

$$
(f \cdot \mathfrak{I}) d_{\mathfrak{z}_{n}}=q^{n} \sum_{\mu} M_{n}\left(\rho_{\mu}^{-1}\right)\left(f d_{\mathfrak{z}_{n}}\right) \circ \rho_{\mu},
$$

$\left\{\rho_{\mu}\right\}$ étant comme plus haut. On peut supposer qu'on a $\rho^{-1} \rho_{\mu} \in G$ pour tout $\mu$. Soit $\mathscr{I}$ un domaine fondamental de $G$. D'après la formule (6), on a

$$
\begin{aligned}
-(2 i)^{n+1}(f \cdot \mathfrak{I}, g) & =q^{n} \sum_{\mu} \int_{\mathscr{F}}\left[\left(f d_{z_{n}}\right) \circ \rho_{\mu}\right]^{t} M_{n}\left(\rho_{\mu}{ }^{-1}\right) P_{n} \overline{g d_{z_{n}}} \\
& =\sum_{\mu} \int_{\rho_{\mu}\left(\mathscr{F}^{\prime}\right)}{ }^{t}\left(f d_{z_{n}}\right) P_{n} M_{n}\left(\rho_{\mu}\right)\left(\overline{g d_{z_{n}}}\right) \circ \rho_{\mu}{ }^{-1} \\
& =\sum_{\mu} \int_{\rho_{\mu}\left(\mathscr{F}^{\prime}\right)}{ }^{t}\left(f d_{z_{n}}\right) P_{n} M_{n}(\rho)\left(\overline{g d_{z_{n}}}\right) \circ \rho^{-1} .
\end{aligned}
$$

Soit $\mathscr{F}_{1}$ un domaine fondamental de $G \cap \rho G \rho^{-1}$; la dernière somme est alors égale à $\int_{\mathscr{F}_{1}}^{t}\left(f d_{\partial_{n}}\right) P_{n} M_{n}(\rho)\left(\overline{g d_{z_{n}}}\right) \circ \rho^{-1}$. On obtient de même, en désignant par $\mathscr{F}_{2}$ un domaine fondamental de $G \cap \rho^{*} G \rho^{*-1}$, 


$$
-(2 i)^{n+1}\left(f, g \cdot \mathfrak{T}^{*}\right)=\int_{\mathscr{T}_{2}}\left[\left(f d_{\mathfrak{z} n}\right) \circ \rho^{*-1}\right]^{t} M_{n}\left(\rho^{*}\right) P_{n} \overline{g d_{z_{n}}} .
$$

Comme on a $G \cap \rho^{*} G \rho^{*-1}=\rho^{-1}\left(G \cap \rho G \rho^{-1}\right) \rho, \rho\left(\mathscr{F}_{2}\right)$ est un domaine fondamental de $G \cap \rho G \rho^{-1}$. On en déduit l'assertion de la proposition.

On va maintenant étudier l'effet de l'opérateur $\mathfrak{I}=\mathfrak{I}(\rho)$ sur les périodes. Soit $f$ un élément de $S_{m}(G)$ et $g=f$. I. Les notations $U, P, M$ étant comme dans le $\S 5$, posons $m=n+2$ et

$$
\omega=\operatorname{Re}\left(U f d_{\mathfrak{z}_{n}}\right), \quad \eta=\operatorname{Re}\left(U g d_{\partial_{n}}\right) .
$$

$\left\{\rho_{\mu}\right\}$ étant comme précédemment, on a

$$
\eta=q^{n} \sum_{\mu} M\left(\rho_{\mu}^{-1}\right) \omega \circ \rho_{\mu},
$$

ou $q=\operatorname{det} \rho$. Définissons les fonctions $\mathfrak{f}, \mathfrak{g}$ et les cocycles paraboliques $\mathfrak{x}, \mathfrak{y}$ comme dans le $\S 4$, avec les constantes d'intégration $\mathfrak{a}, \mathfrak{b}$ et le point de départ $z_{0}$. On a alors, pour chaque $\sigma \in G$,

$$
\begin{aligned}
& \mathfrak{y}(\sigma)-[1-M(\sigma)] \mathfrak{b}=\int_{z_{0}}^{\sigma\left(z_{0}\right)} \eta \\
& =q^{n} \sum_{\mu} M\left(\rho_{\mu}^{-1}\right) \int_{\rho_{\mu}\left(z_{0}\right)}^{\rho_{\mu} \sigma\left(z_{0}\right)} \omega \\
& =q^{n} \sum_{\mu} M\left(\rho_{\mu}^{-1}\right)\left[\mathfrak{f}\left(\rho_{\mu} \sigma\left(z_{0}\right)\right)-\mathfrak{f}\left(\rho_{\mu}\left(z_{0}\right)\right)\right] .
\end{aligned}
$$

D'après notre définition de $\rho_{\mu}$, il existe un indice $\kappa(\mu)$ et un élément $\tau_{\mu}$ de $G$ tels que $\rho_{\mu} \sigma=\tau_{\mu} \rho_{\kappa(\mu)}$; et $\mu \rightarrow \kappa(\mu)$ donne une permutation de $\{1, \cdots, s\}$, où $s$ est le nombre des éléments $\rho_{\mu}$. On a donc

$$
\begin{aligned}
\mathfrak{f}\left(\rho_{\mu} \sigma\left(z_{0}\right)\right) & =\mathfrak{f}\left(\tau_{\mu} \rho_{\kappa(\mu)}\left(z_{0}\right)\right) \\
& =M\left(\tau_{\mu}\right) \mathfrak{f}\left(\rho_{\kappa}(\mu)\left(z_{0}\right)\right)+\mathfrak{x}\left(\tau_{\mu}\right) .
\end{aligned}
$$

Par suite on a

$$
\mathfrak{y}(\sigma)=q^{n} \sum_{\mu} M\left(\rho_{\mu}^{-1}\right) \mathfrak{x}\left(\tau_{\mu}\right)+\mathfrak{r}(\sigma),
$$

où $\mathfrak{r}(\sigma)$ est donné par

$$
\mathfrak{r}(\sigma)=[1-M(\sigma)] \mathfrak{b}+q^{n} \sum_{\mu} M\left(\rho_{\mu}^{-1}\right)\left[M\left(\tau_{\mu}\right) \mathfrak{f}\left(\rho_{\kappa}(\mu)\left(z_{0}\right)\right)-\mathfrak{f}\left(\rho_{\mu}\left(z_{0}\right)\right)\right] .
$$

Posons

$$
\mathfrak{c}=\mathfrak{b}-q^{n} \sum_{\mu} M\left(\rho_{\mu}^{-1}\right) \mathfrak{f}\left(\rho_{\mu}\left(z_{0}\right)\right) .
$$

On a alors, au moyen de la relation $\rho_{\mu}^{-1} \tau_{\mu}=\sigma \rho_{\kappa(\mu)}{ }^{-1}$,

$$
\mathfrak{r}(\sigma)=[1-M(\sigma)] \mathfrak{c} ;
$$

autrement dit, $\mathfrak{r}(\sigma)$ est un cobord. Par conséquent, si l'on pose

$$
\mathrm{t}(\sigma)=q^{n} \sum_{\mu} M\left(\rho_{\mu}^{-1}\right) \mathfrak{x}\left(\tau_{\mu}\right),
$$


$\mathrm{t}(\sigma)$ est un cocycle parabolique et les classes de cohomologie de $\mathfrak{y}$ et de $t$ sont les mêmes. Considérons maintenant le cas où $M_{n}$ satisfait à la condition (A) du $\S 6$. On obtient alors

Théorème 3. Les notations étant comme ci-dessus, supposons que les matrices $P$ et $M(\sigma)$ pour $\sigma \in G$ soient des matrices à coefficients entiers. Si les coefficients de $M\left((\operatorname{det} \rho) \rho^{-1}\right)$ sont entiers, $\mathfrak{I}(\rho)$ donne un endomorphisme de la variété abélienne $S_{m}(G) / D_{m}(G)$.

En effet, on a

$$
q^{n} \sum_{\mu} M\left(\rho_{\mu}^{-1}\right) \mathfrak{x}\left(\tau_{\mu}\right)=\sum_{\mu} M\left(q \rho_{\mu}^{-1}\right) \mathfrak{x}\left(\tau_{\mu}\right) .
$$

On voit que, si $M\left(q \rho^{-1}\right)$ et les $M(\sigma)$ pour $\sigma \in G$ sont à coefficients entiers, il l'est aussi pour les $M\left(\rho_{\mu}\right)$. Par suite, on observe que, si $\mathfrak{x}(\sigma)$ est un élément de $Z(M, \boldsymbol{Z}), \mathrm{t}(\sigma)$ est contenu dans $Z(M, \boldsymbol{Z})$; on en déduit que $\mathfrak{I}(\rho)$ envoie $D_{m}(G)$ dans lui-même; ceci démontre le théorème.

Corollaire. Les notations et les hypothèses étant comme dans le théorème 3 , les racines caractéristiques de $\mathfrak{I}(\rho)$ sont des entiers algébriques de degré $2 h(m)$, où $h(m)$ désigne la dimension de $S_{m}(G)$ sur $C$.

C'est une conséquence immédiate du théorème 3. On voit d'ailleurs, que, si $M(\rho)$ et $M\left(\rho^{*}\right)$ sont tous les deux à coefficients entiers, $\mathfrak{I}(\rho)$ et $\mathfrak{I}\left(\rho^{*}\right)$ sont des endomorphismes de la variété $S_{m}(G) / D_{m}(G)$, et d'après la proposition 2, ils sont les conjugués l'un de l'autre par rapport à la forme de Riemann $c(f, g)$. En particulier, si l'on a $\mathfrak{I}(\rho)=\mathfrak{T}\left(\rho^{*}\right)$, (ce qui est le cas pour les opérateurs de Hecke ordinaires de «Stufe»1), les racines caractéristiques de $\mathfrak{I}(\rho)$ sont des entiers algébriques totalement réels de degré $h(m)$.

\section{§ 9. Exemples.}

On peut appliquer le théorème 2 à tout sous-groupe de $\operatorname{SL}(2, \boldsymbol{Z})$ d'indice fini, puisque $M_{n}(\sigma)$ est à coefficients entiers pour tout $\sigma \in \mathrm{SL}(2, \boldsymbol{Z})$ et tout $n \geqq 0$. Il se trouve donc qu'on obtient une variété abélienne $S_{m}(G) / D_{m}(G)$ pour tout sous-groupe $G$ du groupe $\operatorname{SL}(2, \boldsymbol{Z})$ d'indice fini et pour chaque entier pair positif $m$. Si $\rho$ est un élément de $\mathrm{GL}_{+}(2, \boldsymbol{R})$ à coefficients entiers, $M_{n}(\rho)$ et $M_{n}\left((\operatorname{det} \rho) \rho^{-1}\right)$ sont des matrices à coefficients entiers. Par suite, d'après le théorème 3 , les opérateurs $\mathfrak{I}(\rho), \mathfrak{I}\left(\rho^{*}\right)$ donnent des endomorphismes de la variété abélienne $S_{m}(G) / D_{m}(G)$.

Supposons qu'on ait $\tau^{-1} G \tau=G$ pour un élément $\tau \in \mathrm{SL}(2, \boldsymbol{Z})$. Alors, on obtient un automorphisme $\mathfrak{H}$ de $S_{m}(G)$ en posant

$$
f \cdot \mathfrak{u}=f(\tau(z)) J(\tau, z)^{m}
$$

pour $f \in S_{m}(G)$, et on vérifie de même que pour $\mathfrak{I}(\rho)$ que $\mathfrak{U}$ envoie $D_{m}(G)$ sur $D_{m}(G)$, de sorte que $\mathfrak{U}$ est un automorphisme de $S_{m}(G) / D_{m}(G)$. En particulier, 
si $G$ est un sous-groupe invariant de $\operatorname{SL}(2, \boldsymbol{Z})$ d'indice fini, tout élément de $\mathrm{SL}(2, \boldsymbol{Z})$ engendre ainsi un automorphisme de $S_{m}(G) / D_{m}(G)$. Considérons par exemple le cas où $G$ est le groupe $G(3)$ formé des éléments $\sigma$ de $\operatorname{SL}(2, \boldsymbol{Z})$ tels que $\sigma \equiv I_{2}(\bmod .3)$. On sait que $S_{4}(G(3))$ est de dimension 1 , et est engendré par $\Delta(z)^{1 / 3}$, où $\Delta(z)$ désigne le discriminant de fonctions elliptiques. On voit que $\left(\begin{array}{ll}1 & 1 \\ 0 & 1\end{array}\right)$ donne un automorphisme d'ordre 3 sur $S_{4}(G(3)) / D_{4}(G(3))$. On en déduit que la variété abélienne $S_{4}(G(3)) / D_{4}(G(3))$ est une courbe elliptique à multiplications complexes. Il semble que ce résultat se rattache au fait que la série de Dirichlet correspondant à $\Delta(z)^{1 / 3}$ est le produit de deux fonctions $L$ attachées à des «Grössencharakteren» du corps $\boldsymbol{Q}\left(e^{2 \pi i / 3}\right)$. Plus généralement, quel que soit le sous-groupe $G$ de $\operatorname{SL}(2, \boldsymbol{Z})$, et même s'il n'y a pas de multiplications complexes, on a le droit de penser qu'il existe une relation profonde entre l'arithmétique des séries de Dirichlet attachées aux formes automorphes et nos variétés abéliennes $S_{m}(G) / D_{m}(G)$.

Considérons maintenant le cas où $G=\operatorname{SL}(2, \boldsymbol{Z})$; posons $G(1)=\mathrm{SL}(2, \boldsymbol{Z})$; $G(1)$ est engendré par deux éléments

$$
\sigma=\left(\begin{array}{rr}
0 & 1 \\
-1 & 0
\end{array}\right), \quad \tau=\left(\begin{array}{ll}
1 & 1 \\
0 & 1
\end{array}\right)
$$

Comme on a vu au $\S 3$, on peut prendre $H\left(M_{n}, \boldsymbol{R}, \tau\right)$ au lieu de $H\left(M_{n}, \boldsymbol{R}\right)$; et $H\left(M_{n}, \boldsymbol{Z}, \tau\right)$ s'identifie avec un sous-module de $H\left(M_{n}, \boldsymbol{Z}\right)$ d'indice fini. Désignons par $D_{m}{ }^{\prime}(G(1))$ le sous-module de $D_{m}(G(1))$ correspondant à $H\left(M_{m-2}, \boldsymbol{Z}, \tau\right)$. Alors, $S_{m}(G(1)) / D_{m}{ }^{\prime}(G(1))$ est une variété abélienne isogène à $S_{m}(G(1)) / D_{m}(G(1))$. Tout élément $\mathfrak{x}$ de $Z\left(M_{n}, \boldsymbol{R}, \tau\right)$ est déterminé par un seul vecteur $\mathfrak{x}(\sigma)$. Posons

$$
\begin{aligned}
V_{n} & =\left\{\mathfrak{x}(\sigma) \mid \mathfrak{x} \in Z\left(M_{n}, \boldsymbol{R}, \tau\right)\right\}, \\
W_{n} & =\left\{\mathfrak{x}(\sigma) \mid \mathfrak{x} \in B\left(M_{n}, \boldsymbol{R}, \tau\right)\right\} .
\end{aligned}
$$

On voit facilement que $V_{n}$ est le sous-espace de $\boldsymbol{R}^{n+1}$ formé des vecteurs $\mathfrak{x}$ tels que l'on ait

$$
\begin{gathered}
{\left[1+M_{n}(\sigma)\right] \mathfrak{x}=0,} \\
{\left[1+M_{n}(\sigma \tau)+M_{n}(\sigma \tau)^{2}\right] \mathfrak{x}=0,}
\end{gathered}
$$

et $W_{n}$ est formé des vecteurs $\left[1-M_{n}(\sigma)\right] \mathfrak{y}, \mathfrak{y}$ étant un vecteur tel que

$$
\left[1-M_{n}(\tau)\right] \mathfrak{y}=0
$$

D'après notre définition, $V_{n} / W_{n}$ est canoniquement isomorphe à $S_{n+2}(G(1))$. On vérifie, par un calcul simple, que la forme alternée $\Lambda(f, g)$ sur $S_{n+2}(G(1))$ correspond à la forme bilinéaire $A(\mathfrak{a}, \mathfrak{b})$ sur $V_{n} / W_{n}$ donné par

$$
A(\mathfrak{a}, \mathfrak{b})=c^{t} \mathfrak{a}\left[P_{n} M_{n}(\sigma \tau)-M_{n}(\sigma \tau) P_{n}\right] \mathfrak{b},
$$

où $c$ est un nombre rationnel. 
On va maintenant s'occuper de $S_{12}(G(1)) ; S_{12}(G(1))$ est de dimension 1 et engendré par la fonction $\Delta$ :

Posons

$$
\Delta(z)=q \prod_{n=1}^{\infty}\left(1-q^{n}\right)^{24}: \quad q=e^{2 \pi i z} .
$$

$$
\mathfrak{h}(z)=\int_{\infty}^{z} \Delta(z) d \mathfrak{z}_{10} \cdot
$$

On a alors, pour chaque $r \in G(1)$,

$$
\mathfrak{h}(r(z))=M_{10}(r) \mathfrak{h}(z)+\mathfrak{w}(r),
$$

où $\mathfrak{w}(\gamma)$ est un cocycle parabolique à coefficients complexes. On a evidemment $\mathfrak{w}(\tau)=0$. On obtient, compte tenu de $\sigma(0)=\infty$,

$$
\mathfrak{w}(\sigma)=-\int_{0}^{\infty} \Delta(z) d \mathfrak{z}_{10},
$$

de sorte que les composantes de $\mathfrak{w}(\sigma)$ sont données par

$$
i^{1-\lambda} \int_{0}^{\infty} \Delta(i y) y^{10-\lambda} d y \quad(0 \leqq \lambda \leqq 10) .
$$

Posons $\Delta(z)=\sum_{n=1}^{\infty} a_{n} q^{n}, \Phi(s)=\sum_{n=1}^{\infty} a_{n} n^{-s}$ et $R(s)=(2 \pi)^{-s} \Gamma(s) \Phi(s)$; on a alors

$$
R(s)=\int_{0}^{\infty} \Delta(i y) y^{s-1} d y .
$$

On sait que $R(s)$ est une fonction entière satisfaisant à l'équation fonctionnelle $R(s)=R(12-s)$. D'après (23), les six premières composantes de $\mathfrak{w}(\sigma)$ sont égales à

$$
i R(11), R(10),-i R(9),-R(8), i R(7), R(6) \text {. }
$$

Comme $\mathfrak{w}(\sigma)$ satisfait à (22) et $\left(22^{\prime}\right)$ pour $n=10$, on a

$$
R(10)=\frac{12}{5} R(6), R(8)=\frac{5}{4} R(6), R(9)=\frac{14}{9} R(7) .
$$

Cette relation est analogue à une relation bien connue pour les valeurs de la fonction $\zeta$ de Riemann en les nombres entiers pairs positifs.

Or, on voit que $20 R(6)^{-1} \Delta$ et $9 R(7)^{-1} i \Delta$ engendrent $D_{12}{ }^{\prime}(G(1))$. Par suite, la courbe elliptique $S_{12}(G(1)) / D_{12}{ }^{\prime}(G(1))$ a le module (analytique)

$$
\frac{9}{20} \frac{R(6)}{R(7)} i \text {. }
$$

On peut aborder de même les cas des formes de degré $>12$ ou de «Stufe» $>1$; cependant un calcul pareil ne nous offre rien sur le caractère algébrogéométrique de la variété $S_{m}(G) / D_{m}(G)$.

Nous terminons cette étude en signalant qu'il existe une plus large 
classe de groupes qui satisfont à la condition (A); c'est la classe des groupes fuchsiens attachés aux formes quadratiques ternaires à coefficients entiers (Poincaré [5], Fricke et Klein [2, p. 502]), parmi lesquels le groupe modulaire $\mathrm{SL}(2, \boldsymbol{Z})$ se trouve comme cas particulier.

Université de Tokyo.

\section{Bibliographie}

[1] M. Eichler, Eine Verallgemeinerung der Abelschen Integrale, Math. Z., 67 (1957), 267-298.

[2] R. Fricke et F. Klein, Vorlesungen über die Theorie der Automorphen Funktionen I, Leipzig, 1897.

[3] E. Hecke, Grundlagen einer Theorie der Integralgruppen und der Integralperioden bei den Normalteilern der Modulgruppen, Math. Ann., 116 (1938), 469510.

[4] H. Petersson, Konstruktion der sämtlichen Lösungen einer Riemannschen Funktionalgleichung durch Dirichletreihen mit Eulerscher Produktentwicklung I, Math. Ann., 116 (1938), 401-412.

[5] H. Poincaré, Les fonctions fuchsiennes et l'arithmétique, œuvres, t. II (1887), 463-511.

[6] A. Weil, Généralisation des fonctions abéliennes, J. Math. pures appl., IX. sér. 17 (1938), 47-87.

[7] A. Weil, Variétés kählériennes, Hermann, Paris, 1958.

[8] A. Weil, Sur les courbes algébriques et les variétés qui s'en déduisent, Hermann, Paris, 1948. 